\title{
Cloning, characterization, and expression of the macrophage migration inhibitory factor gene from the Pacific white shrimp Litopenaeus vannamei (Penaeidae)
}

\author{
D.G. Zeng*, A.Y. Lei* and X.H. Chen \\ Guangxi Key Laboratory of Aquatic Genetic Breeding and Healthy Aquaculture, \\ Guangxi Institute of Fisheries, Nanning, China \\ *These authors contributed equally to this study. \\ Corresponding author: X.H. Chen \\ E-mail: gxnnscs@126.com
}

Genet. Mol. Res. 12 (4): 5872-5879 (2013)

Received March 8, 2013

Accepted July 1, 2013

Published November 22, 2013

DOI http://dx.doi.org/10.4238/2013.November.22.15

\begin{abstract}
The macrophage migration inhibitory factor (MIF) is an important proinflammatory cytokine that mediates both innate and adaptive immune responses. In this study, we identified a homolog of MIF in the Pacific white shrimp Litopenaeus vannamei. The MIF cDNA contained a 363 -bp open reading frame encoding a 120-amino acid protein with a calculated molecular mass of $13.442 \mathrm{kDa}$ and a theoretical isoelectric point of 6.57. The L. vannamei MIF shared high amino acid identity with MIFs of other invertebrates. Tissue distribution analysis by quantitative real-time polymerase chain reaction (qRT-PCR) revealed that the L. vannamei MIF was abundantly expressed in the blood, heart, and hepatopancreas, was moderately expressed in the gill, and was weakly expressed in the muscle and intestine. Furthermore, in order to gain a basic understanding of the role of MIF in the shrimp immune response against viral infection, its mRNA expression was determined in the hepatopancreas of $L$. vannamei at different stages after white
\end{abstract}


spot syndrome virus (WSSV) challenge using qRT-PCR. The result indicated that the expression of MIF was significantly upregulated after WSSV injection, suggesting that MIF may be involved in the response to viral infection in shrimp.

Key words: Macrophage migration inhibitory factor; Cloning; Litopenaeus vannamei

\section{INTRODUCTION}

The Pacific white shrimp, Litopenaeus vannamei (Lee, 1931), has become the leading farm crustacean species in the world (Valles-Jimenez et al., 2004). Because of its huge economic value and important evolutionary status, an increasing number of studies have focused on the biology, physiology, genetics, and immunology of L. vannamei in recent years (Shen et al., 2007; Ciobanu et al., 2010; Gorbach et al., 2010).

The macrophage migration inhibitory factor (MIF) is one of the earliest discovered lymphocyte factors (Bloom and Bennett, 1966). MIF was originally identified as an immunoregulatory cytokine produced by antigen-activated $\mathrm{T}$ lymphocytes that inhibited the random migration of macrophages (Bacher et al., 1996). Recent research has revealed a more prominent role of MIF as a multi-functional cytokine that is involved in cell-mediated immunity, immunoregulation, and inflammation (Larson and Horak, 2006; Flaster et al., 2007; Stojanovic et al., 2011). Further research has shown that MIF plays a crucial role in malignant disease progression (Chuang et al., 2011; Herrero et al., 2011; Yeh et al., 2013). Since the human MIF gene was first cloned (Weiser et al., 1989), MIF homologs have been identified in various vertebrate and invertebrate species (Mitchell et al., 1995; Jin et al., 2007; Lopes et al., 2011). However, to our knowledge, MIF has not yet been cloned in shrimp.

Shrimp lack an acquired immune system, and must instead rely on efficient innate immune defenses (Yeh et al., 2009). Therefore, an understanding of the innate immune ability of shrimp and their defense mechanisms is becoming an increasingly important research subject (Sivakamavalli and Vaseeharan, 2012). In order to determine the participation of MIF in the innate immune response in shrimp, we identified a cDNA encoding MIF from L. vannamei, and analyzed its characteristics, evolution, tissue distribution, and differential expression in response to viral infection.

\section{MATERIAL AND METHODS}

\section{L. vannamei MIF cloning and sequence analysis}

Construction of a cDNA library from the L. vannamei hepatopancreas and sequencing of expressed sequence tags (EST) were previously completed in our research group. A cDNA containing the complete amino acid sequence of MIF was obtained by searching against similar sequences in the National Center for Biotechnology Information (NCBI) non-redundant (nr) protein database using the BLASTX programs (http://blast.ncbi.nlm.nih.gov/Blast.cgi). The NCBI open reading frame (ORF) Finder program (http://www.ncbi.nlm.nih.gov/projects/ gorf/) was used to identify the MIF cDNA ORF and the DNA translation. The theoretical iso- 
electric point ( $\mathrm{pI}$ ) and the molecular weight $(\mathrm{Mw})$ of the MIF were computed using the $\mathrm{pI} / \mathrm{Mw}$ tool (http://web.expasy.org/compute_pi/). The secondary structure of the MIF was predicted using the PredictProtein tool (https://www.predictprotein.org). Multiple sequence alignment was conducted using the ClustalW2 software (http://www.ebi.ac.uk/Tools/msa/clustalw2/), and a phylogenetic tree was created using the MEGA5.1 software (http://www.megasoftware. net/) by the "neighbor-joining" method and was bootstrapped 2000 times.

\section{Experimental shrimp, white spot virus syndrome (WSSV) challenge, and quantitative real-time polymerase chain reaction (qRT-PCR) analysis}

Specific pathogen-free (SPF) L. vannamei shrimp were obtained from the National and Guangxi Shrimp Genetic Breeding Center (Guangxi Province, China). The average body weight of the shrimp was approximately $10 \mathrm{~g}$. The shrimp were maintained in $1000-\mathrm{L}$ glass seawater tanks $\left(25^{\circ}\right.$ to $\left.26^{\circ} \mathrm{C}\right)$ and fed an artificial pellet feed. The shrimp were allowed to acclimatize for 2 weeks before the experiment. For the experiment, the shrimp were divided into two groups: The WSSV challenge group and the normal control group. Shrimp in the challenge group were injected with a $60-\mu \mathrm{L}$ WSSV suspension prepared as described by Prior et al. (2003). In parallel, the shrimp in the control group were injected with a $60-\mu \mathrm{L}$ extract prepared from a healthy shrimp. The shrimp were randomly collected at $0,12,24,48$, and $72 \mathrm{~h}$ postinjection. At each time point, the hepatopancreas was isolated from three individuals in each group and frozen in liquid nitrogen. Total RNA was extracted using TRIzol reagent (Qiagen) following manufacturer instructions, and DNA was removed with DNase (Promega). The RNA was stored at $-80^{\circ} \mathrm{C}$ until use. The primers $5^{\prime}$-TTAGTGAAACCATTGGGAAA-3' (forward) and 5'-AACTGCATAAATCTTAGAAG-3' (reverse) for qRT-PCR were designed according to the MIF gene sequence. The primers for $\beta$-actin (used as an internal reference gene) were 5'-CGAGAAATCGTTCGTGAC-3' (forward) and 5'-GATGGAGTTGTAGGTGGTCT-3' (reverse). The first-strand cDNA was synthesized from $1 \mu \mathrm{g}$ RNA with M-MuLV reverse transcriptase (Qiagen). The 20- $\mu \mathrm{L}$ qPCR reaction mixture consisted of $2 \mathrm{X}$ Power SYBR Green PCR Master mix, $0.9 \mathrm{M}$ each forward and reverse primers, and $1 \mu \mathrm{L}$ template cDNA. PCR amplification was performed under the following program: $50^{\circ} \mathrm{C}$ for $2 \mathrm{~min}$ and $95^{\circ} \mathrm{C}$ for $30 \mathrm{~s} ; 35$ cycles of $95^{\circ} \mathrm{C}$ for $15 \mathrm{~s}$ and $62^{\circ} \mathrm{C}$ for $1 \mathrm{~min} ; 72^{\circ} \mathrm{C}$ for $5 \mathrm{~min}$.

\section{RESULTS}

\section{Cloning and sequence analysis of the MIF gene}

A cDNA containing the complete amino acid sequence of MIF was obtained by sequencing the $L$. vannamei cDNA library. The cDNA sequence has been submitted to GenBank under the accession No. KC513658. The cDNA contained a 363-bp ORF encoding a 120-amino acid protein with a calculated $\mathrm{Mw}$ of $13.442 \mathrm{kDa}$ and a $\mathrm{pI}$ of 6.57 . To determine the protein family, the structural domains and functional sites of the MIF polypeptide sequence were analyzed by searching the Pfam database. The results showed that the MIF amino acid sequence contained a Pfam profile CL0082 (MIF), indicating that the L. vannamei MIF identified in this study belongs to the MIF protein family. Furthermore, secondary structure prediction indicated that the MIF contained three alpha helices and five beta sheets in accordance with the 
$\beta 1 \alpha 1 \beta 2 \beta 3 \beta 4 \alpha 2 \beta 5 \alpha 3$ order (Figure 1). The signal peptide cleavage site was predicted using the SignalP3.0 program. The result showed that the peptide of MIF did not contain a signal peptide sequence, implying that it is not released from the cells by the conventional protein secretory pathway.

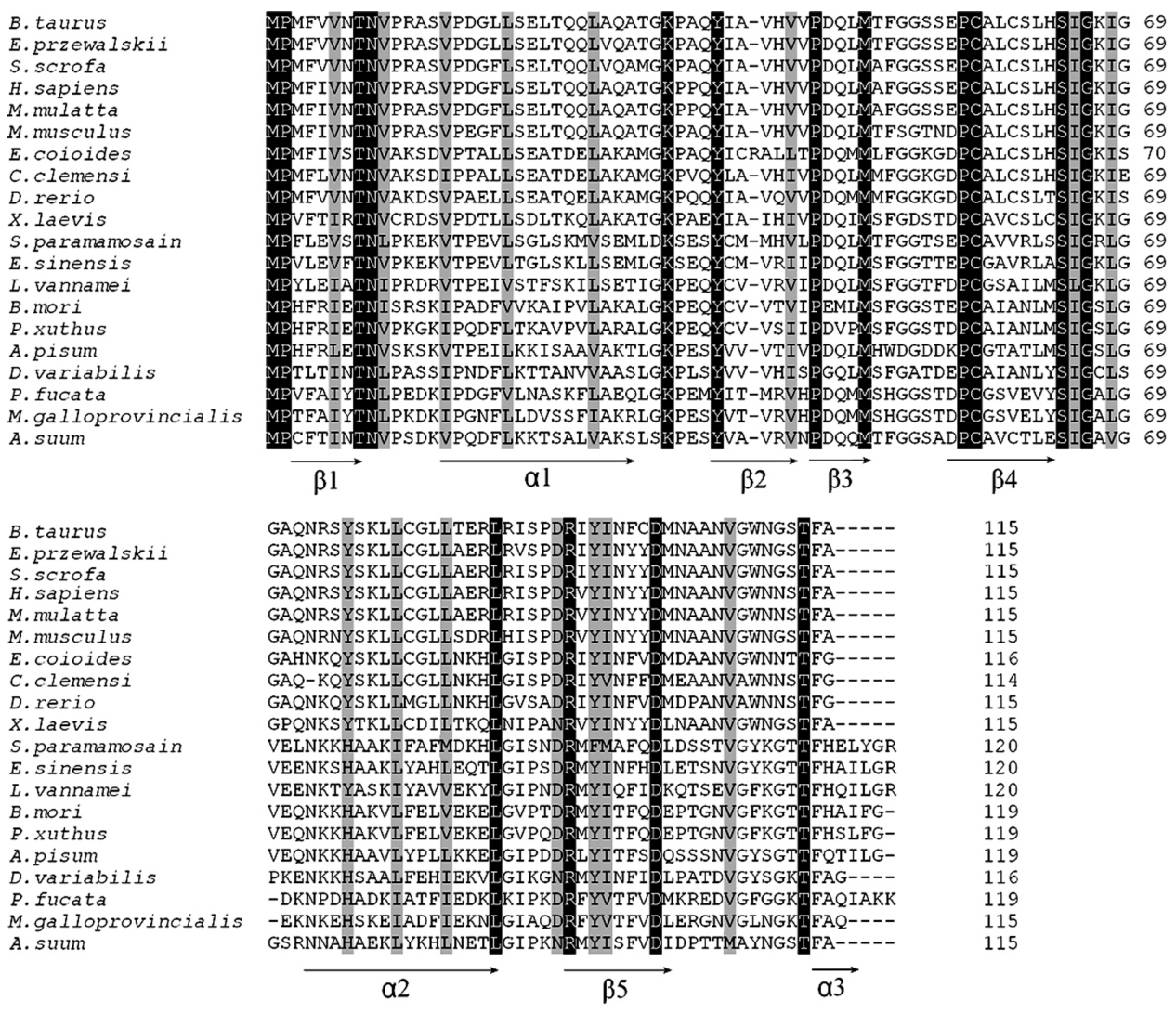

Figure 1. Multiple sequence alignment of migration inhibitory factor (MIF) amino acid sequences. The reported MIF amino acid sequences were from GenBank and the accession numbers are as follows: Scylla paramamosain (AFU81798), Eriocheir sinensis (ADM86239), Bombyx mori (ABD36229), Papilio xuthus (BAM18142), Acyrthosiphon pisum (XP_003247825), Pinctada fucata (ADU19847), Danio rerio (NP_001036786), Ascaris suum (BAD24819), Bos taurus (NP_001028780), Homo sapiens (AAA36315), Sus scrofa (ABI95381), Mus musculus (AAA74321), Macaca mulatta (NP_001028087), Mytilus galloprovincialis (AEN25591), Epinephelus coioides (AEA39742), Caligus clemensi (ACŌ15094), Dermacentor variabilis (ACF35547), Equus przewalskii (ACL36425).

The full lengths of the MIF protein and its homologs in other species were subjected to phylogenetic analysis using ClustalW2, and a phylogenetic tree was created using the MEGA5.1 software. According to the phylogenetic tree (Figure 2), the L. vannamei MIF was clustered to a subtree followed by the branches of invertebrate MIF proteins. L. vannamei MIF mostly clustered with the MIF of Scylla paramamosain and Eriocheir sinensis, which also belong to the crustacean family. 


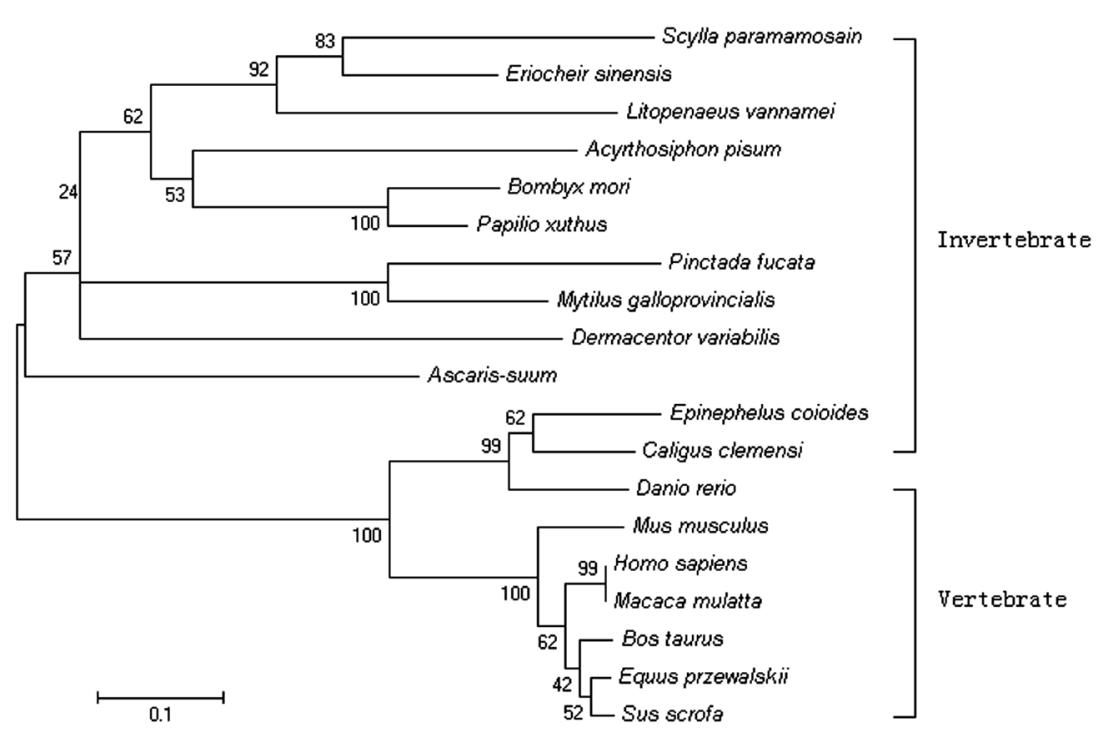

Figure 2. Phylogenetic tree showing the relationship between Litopenaeus vannamei MIF sequence with other known MIF proteins. The reported MIF amino acid sequences were from GenBank and the accession numbers are as follows: Scylla paramamosain (AFU81798), Eriocheir sinensis (ADM86239), Bombyx mori (ABD36229), Papilio xuthus (BAM18142), Acyrthosiphon pisum (XP_003247825), Pinctada fucata (ADU19847), Danio rerio (NP 001036786), Ascaris suum (BAD24819), Bos taurus (NP_001028780), Homo sapiens (AAA36315), Sus scrofa (ABI95381), Mus musculus (AAA74321), Macaca mulatta (NP_001028087), Mytilus galloprovincialis (AEN25591), Epinephelus coioides (AEA39742), Caligus clemensi (ACO15094), Dermacentor variabilis (ACF35547), Equus przewalskii (ACL36425). Numbers on the branches represent bootstrap support for 2000 replicates. The scale bar represents a genetic distance of 0.1 .

\section{Tissue expression of MIF}

MIF expression was determined in various tissues of $L$. vannamei before WSSV challenge using qRT-PCR. $\beta$-actin of $L$. vannamei was used as a housekeeping control gene, and the intestine was used as a calibration sample for RNA amount standardization. The results of qRT-PCR showed that MIF was expressed in the intestine, muscle, gill, hepatopancreas, heart, and blood (Figure 3). In addition, MIF transcript amounts differed in various tissues, with the highest expression in the hepatopancreas and the lowest in the muscle (Figure 4).

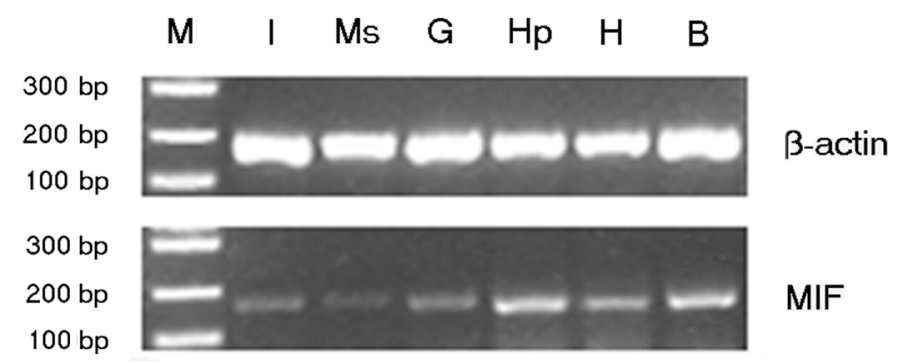

Figure 3. RT-PCR analysis of migration inhibitory factor (MIF) expression in various tissues, including intestine $(\mathrm{I})$, muscle $(\mathrm{Ms})$, gill $(\mathrm{G})$, hepatopancreas $(\mathrm{Hp})$, heart $(\mathrm{H})$, and blood $(\mathrm{B})$ from normal shrimp. $\beta$-actin was used as the internal control for all tissues. Lane $M=$ molecular weight marker. 


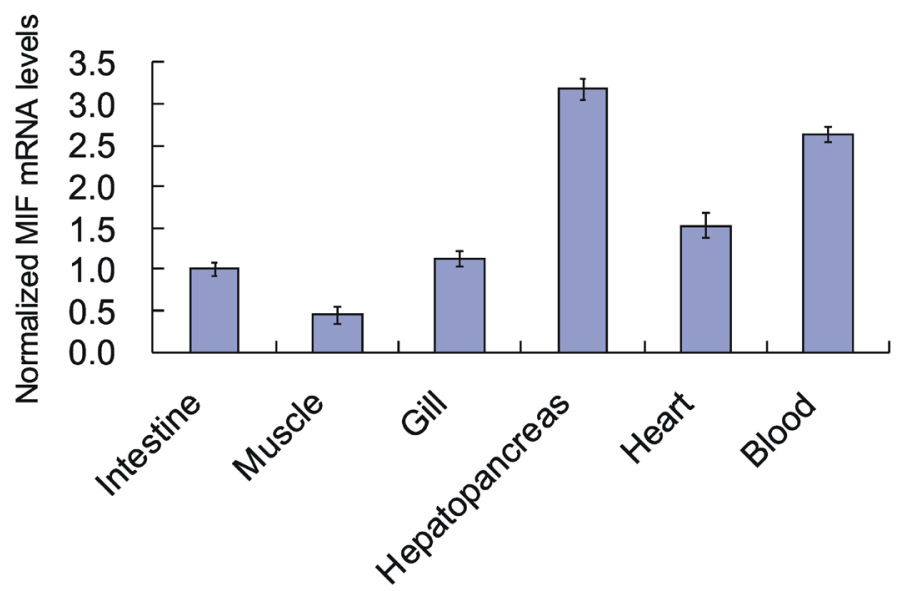

Figure 4. Real-time quantitative RT-PCR analysis of migration inhibitory factor (MIF) expression in various tissues.

\section{Expression of MIF after WSSV challenge}

The hepatopancreases were collected at $0,6,12,24$, and $48 \mathrm{~h}$ after WSSV challenge. The expression of MIF was analyzed by qRT-PCR. The results showed an obvious increase in the level of MIF transcripts. The expression was highest at $12 \mathrm{~h}$ and decreased gradually at later time points. Nonetheless, the expression of MIF was significantly up-regulated during the WSSV-infected phase relative to that of the unchallenged control shrimp (Figure 5).

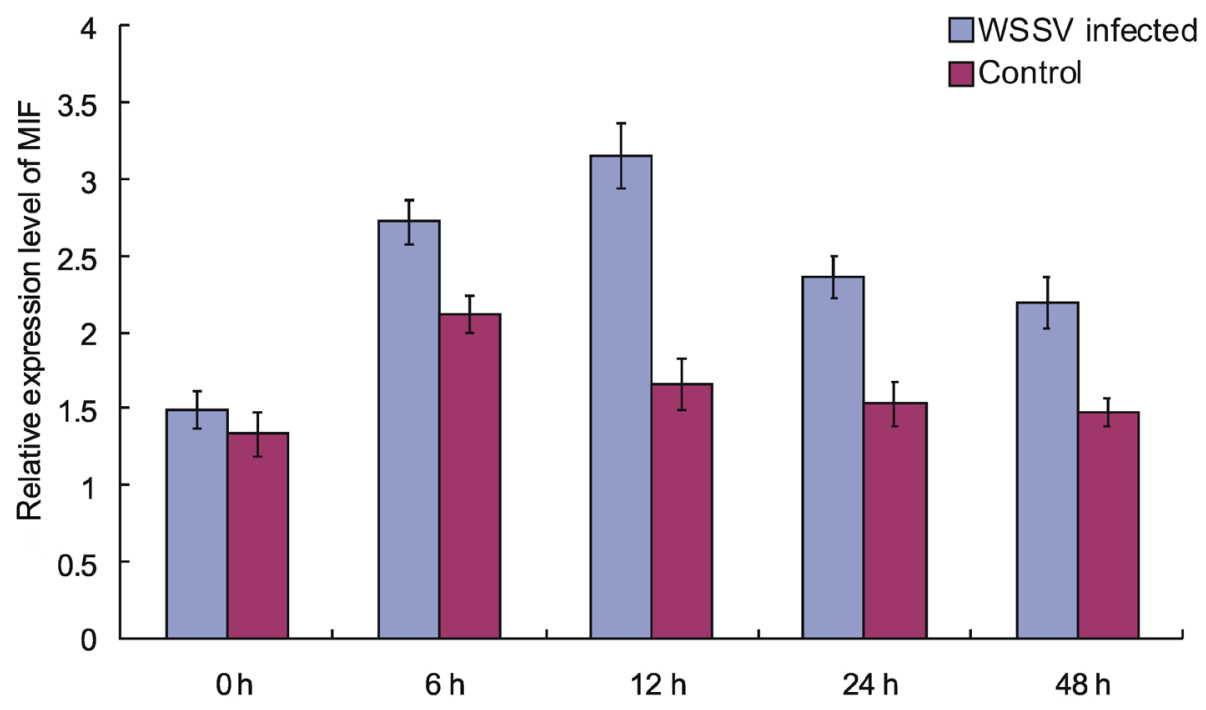

Figure 5. Real-time quantitative RT-PCR analysis of the migration inhibitory factor (MIF) expression in hepatopancreas of Litopenaeus vannamei after white spot syndrome virus (WSSV) challenge. The x-axis shows hours post-WSSV challenge and the y-axis shows the relative expression level of MIF (means $\pm 2 \mathrm{SE}, \mathrm{N}=3$ ). 


\section{DISCUSSION}

Cytokines play a crucial role in the immune response by regulating both innate and acquired immunity (Stenger and Rollinghoff, 2001). Numerous studies have indicated that these molecules are implicated in many human pathological conditions (Sládková and Kostolanský, 2006; Mustafa et al., 2007; Linker et al., 2008). However, relatively few cytokines have been identified in crustaceans, and in shrimp in particular, to date. In this study, we cloned, for the first time, the MIF from $L$. vannamei. Amino acid sequences and phylogenetic analysis revealed close similarity between $L$. vannamei MIF and other invertebrate MIF proteins. The phylogenetic analysis of MIF sequences coincides with current understanding of animal evolution. Secondary structure prediction indicated that $L$. vannamei MIF possesses three alpha helices and five beta sheets in accordance with the $\beta 1 \alpha 1 \beta 2 \beta 3 \beta 4 \alpha 2 \beta 5 \alpha 3$ order, which is similar to human MIF, whose secondary structure is comprised of two alpha helices and six beta sheets in the following order: $\beta 1 \alpha 1 \beta 2 \beta 3 \beta 4 \alpha 2 \beta 5 \beta 6$ (Sun et al., 1996). This implies that the biological functions of $L$. vannamei MIF may be similar to those of human MIF.

Consistent with previous studies showing that MIF is ubiquitously expressed in both immune and non-immune cells of various tissues (Jian and Parker Jr., 2003), in the present study, qRT-PCR analysis showed that the MIF was constitutively expressed in six selected tissues from healthy $L$. vannamei individuals. The expression level of $L$. vannamei MIF was higher in the hepatopancreas than those in the intestine, muscle, gill, heart, and blood. Since the hepatopancreas is considered to play a major role in initiating the defense response in shrimp (Ji et al., 2009; Li and Brouwer, 2013), the higher expression of MIF in the hepatopancreas suggested that MIF is involved in the immune response of shrimp. Furthermore, expression profiles of MIF in the hepatopancreas of L. vannamei at different interval times following WSSV challenge showed a significant increase in the expression level of MIF, indicating that MIF might participate in the shrimp immune response to viral infection. However, the molecular mechanism of MIF action remains to be investigated in more depth.

In conclusion, we cloned the MIF cDNA from L. vannamei. The molecular and structural characterization of MIF will help to understand the immune system of shrimp.

\section{ACKNOWLEDGMENTS}

Research supported by the Natural Science Foundation of China (\#31160531), the "Twelfth Five-Year" National Science and Technology Project in Rural Areas (\#2012AA10A404-3), and the Guangxi Department of Science (\#11107012-1-2).

\section{REFERENCES}

Bacher M, Metz CN, Calandra T, Mayer K, et al. (1996). An essential regulatory role for macrophage migration inhibitory factor in T-cell activation. Proc. Natl. Acad. Sci. U. S. A. 93: 7849-7854.

Bloom BR and Bennett B (1966). Mechanism of a reaction in vitro associated with delayed-type hypersensitivity. Science 153: 80-82.

Chuang YC, Lei HY, Liu HS, Lin YS, et al. (2011). Macrophage migration inhibitory factor induced by dengue virus infection increases vascular permeability. Cytokine 54: 222-231.

Ciobanu DC, Bastiaansen JW, Magrin J, Rocha JL, et al. (2010). A major SNP resource for dissection of phenotypic and genetic variation in Pacific white shrimp (Litopenaeus vannamei). Anim. Genet. 41: 39-47. 
Flaster H, Bernhagen J, Calandra T and Bucala R (2007). The macrophage migration inhibitory factor-glucocorticoid dyad: regulation of inflammation and immunity. Mol. Endocrinol. 21: 1267-1280.

Gorbach DM, Hu ZL, Du ZQ and Rothschild MF (2010). Mining ESTs to determine the usefulness of SNPs across shrimp species. Anim. Biotechnol. 21: 100-103.

Herrero LJ, Nelson M, Srikiatkhachorn A, Gu R, et al. (2011). Critical role for macrophage migration inhibitory factor (MIF) in Ross River virus-induced arthritis and myositis. Proc. Natl. Acad. Sci. U. S. A. 108: 12048-12053.

Ji PF, Yao CL and Wang ZY (2009). Immune response and gene expression in shrimp (Litopenaeus vannamei) hemocytes and hepatopancreas against some pathogen-associated molecular patterns. Fish Shellfish Immunol. 27: 563-570.

Jian M and Parker CR Jr (2003). Macrophage migration inhibitory factor is a constitutively expressed cytokine in the human adrenal gland. IUBMB Life 55: 155-158.

Jin HJ, Xiang LX and Shao JZ (2007). Molecular cloning and identification of macrophage migration inhibitory factor (MIF) in teleost fish. Dev. Comp. Immunol. 31: 1131-1144.

Larson DF and Horak K (2006). Macrophage migration inhibitory factor: controller of systemic inflammation. Crit. Care 10: 138 .

Lee B (1931). Anomuran, macruran Crustacea from Panama and Canal Zone. Bull. Am. Mus. Nat. Hist. 63: 137-189.

$\mathrm{Li}$ T and Brouwer M (2013). Gene expression profile of hepatopancreas from grass shrimp Palaemonetes pugio exposed to cyclic hypoxia. Comp. Biochem. Physiol. Part D. Genomics Proteomics 8: 1-10.

Linker RA, Kruse N, Israel S, Wei T, et al. (2008). Leukemia inhibitory factor deficiency modulates the immune response and limits autoimmune demyelination: a new role for neurotrophic cytokines in neuroinflammation. J. Immunol. 180: 2204-2213.

Lopes F, Vannoni A, Sestini S, Casciaro A, et al. (2011). Sheep (Ovis aries) Macrophage Migration Inhibitory Factor: molecular cloning, characterization, tissue distribution and expression in the ewe reproductive tract and in the placenta. Cytokine 54: 315-323.

Mitchell R, Bacher M, Bernhagen J, Pushkarskaya T, et al. (1995). Cloning and characterization of the gene for mouse macrophage migration inhibitory factor (MIF). J. Immunol. 154: 3863-3870.

Mustafa T, Wiker HG, Morkve O and Sviland L (2007). Reduced apoptosis and increased inflammatory cytokines in granulomas caused by tuberculous compared to non-tuberculous mycobacteria: role of MPT64 antigen in apoptosis and immune response. Clin. Exp. Immunol. 150: 105-113.

Prior S, Browdy CL, Shepard EF, Laramore R, et al. (2003). Controlled bioassay systems for determination of lethal infective doses of tissue homogenates containing Taura syndrome or white spot syndrome virus. Dis. Aquat. Organ 54: 89-96.

Shen X, Ren J, Cui Z, Sha Z, et al. (2007). The complete mitochondrial genomes of two common shrimps (Litopenaeus vannamei and Fenneropenaeus chinensis) and their phylogenomic considerations. Gene 403: 98-109.

Sivakamavalli JRP and Vaseeharan B (2012). Prophenoloxidase and immune indices of Indian White Shrimp Fenneropenaeus indicus. J. Aquacult. Res. Dev. 3: e1000148.

Sládková T and Kostolanský F (2006). The role of cytokines in the immune response to influenza A virus infection. Acta Virol. 50: 151-162.

Stenger S and Rollinghoff M (2001). Role of cytokines in the innate immune response to intracellular pathogens. Ann. Rheum. Dis. 60 (Suppl 3): iii43-iii46.

Stojanovic I, Mirkov I, Kataranovski M, Glamoclija J, et al. (2011). A role for macrophage migration inhibitory factor in protective immunity against Aspergillus fumigatus. Immunobiology 216: 1018-1027.

Sun HW, Bernhagen J, Bucala R and Lolis E (1996). Crystal structure at 2.6-A resolution of human macrophage migration inhibitory factor. Proc. Natl. Acad. Sci. U. S. A. 93: 5191-5196.

Valles-Jimenez R, Cruz P and Perez-Enriquez R (2004). Population genetic structure of Pacific white shrimp (Litopenaeus vannamei) from Mexico to Panama: microsatellite DNA variation. Mar. Biotechnol. 6: 475-484.

Weiser WY, Temple PA, Witek-Giannotti JS, Remold HG, et al. (1989). Molecular cloning of a cDNA encoding a human macrophage migration inhibitory factor. Proc. Natl. Acad. Sci. U. S. A. 86: 7522-7526.

Yeh SP, Chen YN, Hsieh SL, Cheng W, et al. (2009). Immune response of white shrimp, Litopenaeus vannamei, after a concurrent infection with white spot syndrome virus and infectious hypodermal and hematopoietic necrosis virus. Fish Shellfish Immunol. 26: 582-588.

Yeh TM, Liu SH, Lin KC, Kuo C, et al. (2013). Dengue virus enhances thrombomodulin and ICAM-1 expression through the macrophage migration inhibitory factor induction of the MAPK and PI3K signaling pathways. PLoS One 8: e55018. 